\title{
Group formation through indirect reciprocity
}

\author{
Koji Oishi, ${ }^{1,2}$ Takashi Shimada, ${ }^{1,2}$ and Nobuyasu Ito ${ }^{1,2,3}$ \\ ${ }^{1}$ Department of Applied Physics, Graduate School of Engineering, University of Tokyo, 7-3-1 Hongo, Bunkyo-ku, Tokyo, 113-8656, Japan \\ ${ }^{2}$ JST, CREST, 4-1-8 Honcho, Kawaguchi, Saitama, 332-0012, Japan \\ ${ }^{3}$ Discrete Event Simulation Research Team, RIKEN Advanced Institute for Computational Science, 7-1-26 Minatojima-minami-machi, \\ Chuo-ku, Kobe, Hyogo, 650-0047, Japan
}

(Received 30 November 2012; published 6 March 2013)

\begin{abstract}
The emergence of group structure of cooperative relations is studied in an agent-based model. It is proved that specific types of reciprocity norms lead individuals to split into two groups only inside of which they are cooperative. The condition for the evolutionary stability of the norms is also obtained. This result suggests reciprocity norms, which usually promote cooperation, can cause society's separation into multiple groups.
\end{abstract}

DOI: 10.1103/PhysRevE.87.030801

PACS number(s): 89.65.Ef, 87.23.Ge, 02.50.Le, 89.75.Fb

Group formation in human society has attracted much interest in statistical physics as a example of self-organizing phenomena in complex systems [1-4]. Social systems include complex interactions which are unconventional in physics. Especially, a three-body interaction naturally arises from people's ability of monitoring what others do to others. While such complex interactions are expected to bring about various novel phenomena, group formation based on this monitoring ability has not been studied well.

Mutual monitoring is pointed out to be playing a key role for promotion of cooperation in society. A longstanding question in the studies on cooperative behavior is how people can maintain mutual cooperation even when they are faced with a social dilemma, which means the situation that people will fail to cooperate even though entire cooperation gives better payoff to everyone. Several mechanisms have been pointed out to be important to overcome the social dilemma. Reciprocity is one of them [5]. Especially, indirect reciprocity (cooperation to someone enhances his probability to have a cooperative response from others) is a key when partners to interact are flexible [6]. It is necessary for this mechanism that people monitor what others do to others and appropriately find those whom they should cooperate with. Otherwise free-riding behavior spreads and cooperation is abandoned.

An important aspect of cooperation is that people are generally more cooperative to the members of the same group, which is called in-group favoritism [7]. To understand in-group favoritism, one can assume given group structure and study how cooperation is sustained only inside of the groups [8-10]. In some cases, however, the group structure itself can be formed by social interaction of people. For example, groups of friends or business partners are hardly decided a priori. Therefore, it is essential to model group formation and in-group favoritism together.

Here we study whether group structure of cooperation is spontaneously formed via indirect reciprocity with private assessment: people can have different opinions on a person's reputation. Private assessment models themselves have been studied in previous works [11-14]. However the main issue in those works is to find what kind of definition of goodness can evolutionarily promote cooperation, not group formation. In this paper, on the other hand, the structure of the cooperative relations is the main interest.
We consider well-mixed $N$ players playing a game, which is called donation game. The number of players is assumed to be large, $N \gg 1$. The game is repeated over a large number of rounds. In every round, one player is randomly chosen as a donor and another player is randomly chosen as a recipient. The donor decides whether he cooperates or defects. If the donor cooperates, the payoff of the donor in the round is $-c<0$ and that of the recipient is $b>0$. If the donor defects, payoffs of both players are $0 . b$ and $c$ are called benefit of cooperation and cost of cooperation respectively. We assume $b>c$, that means when players always cooperate the average payoff per round $(b-c) / N$ is positive. When they always defect, the average payoff is 0 . Therefore, when all cooperate, all benefit, however one can increase his own payoff by defecting others. In this sense, players in this game are faced with a social dilemma.

Each player has his own opinion on each of all players whether he is good or bad (opinion matrix). The donor of each round decides his action based on his opinion on the recipient and his action rule. All players observe who is the donor, who is the recipient, and what the donor does to the recipient. Then all players independently revise their own opinion on the donor based on the observation and his assessment rule. A round ends after all players revise their opinion. Players do not change their action rule and assessment rule in the repetition of the game. And all players are assumed to adopt a common assessment rule.

$\beta_{i j}(t) \in\{G, B\}$ represents player $i$ 's opinion on player $j$ at the outset of round $t$. If player $i$ has the opinion that player $j$ is good at the outset of round $t$ then $\beta_{i j}(t)=G$ and if $i$ has the opinion that $j$ is bad then $\beta_{i j}(t)=B$. In this paper, the matrix $\beta(t)=\left\{\beta_{i j}(t)\right\}_{i, j}$ is called an opinion matrix, which was called an image matrix in previous works $[13,14]$. Each element of the opinion matrix at the initial round $t=1$, is $G$ with probability $p$ and $B$ with probability $1-p$. The probability $p$ is called initial trust probability and assumed it is not 0 or 1 . Note that each player $i$ also has the opinion on himself $\beta_{i i}$, which can matter when he is the recipient and revises his opinion on the donor.

An action matrix $\gamma(t)=\left\{\gamma_{i j}(t)\right\}_{i \neq j}$ represents the action each player will take to each of other players if they are matched at round $t$. If $i$ will cooperate with $j, \gamma_{i j}(t)=C$ and we say $i$ is cooperative to $j$, while if $i$ will defect against 
TABLE I. Definition of 16 assessment rules (2nd to 5th columns) and corresponding stationary states of the action matrix with each assessment rule and DISC or pDISC action rules (6th and 7th columns). Each element in the 2nd to 5th columns shows the observer's new opinion based on his observation: [recipient's reputation $(G$ or $B)$, donor's action ( $C$ or $D)$ ]. The classification of the state form (a) to (h) is described in the FIG. 1.

\begin{tabular}{|c|c|c|c|c|c|c|}
\hline rule & $(G, C)$ & $(G, D)$ & $(B, C)$ & $(B, D)$ & DISC & pDISC \\
\hline 1 & $B$ & $B$ & $B$ & $B$ & (b) & (a) \\
\hline 2 & $B$ & $B$ & $B$ & $G$ & (e) & (a) \\
\hline 3 & $B$ & $B$ & $G$ & $B$ & (b) & (e) \\
\hline 4 & $B$ & $B$ & $G$ & $G$ & (h) & (h) \\
\hline 5 & $B$ & $G$ & $B$ & $B$ & (b) & (a) \\
\hline 6 & $B$ & $G$ & $B$ & $G$ & (e) & (a) $/(b)$ \\
\hline 7 & $B$ & $G$ & $G$ & $B$ & (g) & (g) \\
\hline 8 & $B$ & $G$ & $G$ & $G$ & (e) & (b) \\
\hline 9 & $G$ & $B$ & $B$ & $B$ & (b) & (a) \\
\hline 10 & $G$ & $B$ & $B$ & $G$ & (f) & (f) \\
\hline 11 & $G$ & $B$ & $G$ & $B$ & (a) $/(\mathrm{b})$ & (e) \\
\hline 12 & $G$ & $B$ & $G$ & $G$ & (a) & (e) \\
\hline 13 & $G$ & $G$ & $B$ & $B$ & (c) & (d) \\
\hline 14 & $G$ & $G$ & $B$ & $G$ & (a) & (b) \\
\hline 15 & $G$ & $G$ & $G$ & $B$ & (a) & (b) \\
\hline 16 & $G$ & $G$ & $G$ & $G$ & (a) & (b) \\
\hline
\end{tabular}

$j, \gamma_{i j}(t)=D$ and we say $i$ is not cooperative to $j$. Diagonal elements are not defined since no one plays the game with himself. We omit the argument $(t)$ when it is not confusing.

The deterministic map from one's opinion on the opponent to the action he will take is called an action rule. Since the number of the possible opinions and that of possible actions are two, the number of the possible action rules are $4=2^{2}$. The four action rules are called DISC, pDISC, All-C, and All-D, and those who adopt each action rule are called DISC players, pDISC players, All-C players, and All-D players, respectively. All-C players always cooperate and All-D players always do not cooperate regardless of their opinion on the recipient. In contrast, DISC players cooperate only when they regard the recipient as good while pDISC players cooperate only with bad recipients in their opinion.

Players also adopt a certain assessment rule, which defines their opinions on the donor after observing a game round. There are $2 \times 2$ possible combinations of an observer's opinion on the recipient and the action of the donor. Therefore, there are $2^{4}$ assessment rules, which are called rule 1 to rule 16 . The left part of Table I shows the definition of the 16 assessment rules.

First, we assume all players adopt a common action rule. Under this restriction, we still have $16 \times 4$ cases for combinations of an assessment rule and an action rule. Although the time development of an action matrices is stochastic and complicated, we have analytically derived the stationary states [15]. All stationary states can be classified into eight distinct states, according to the clustering structure of the action matrix. Figure 1 shows the eight stationary states, which are called states (a) to (h). The sizes of the clusters can be stochastic variable in some states, however we neglect the fluctuation since $N$ is assumed to be very large. If all players

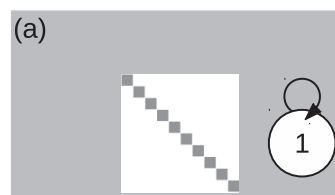

(b)
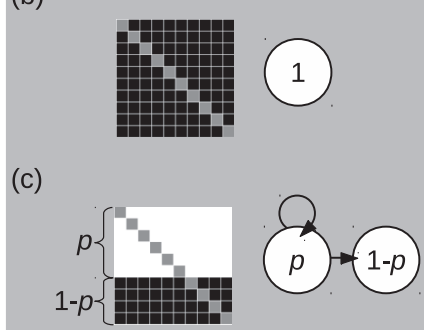

(d)

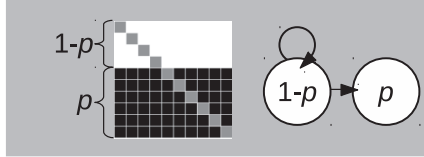

(e)

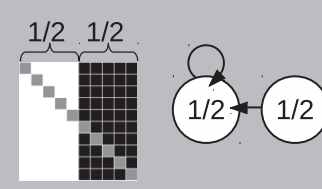

(f)

P 1-p

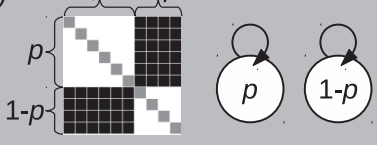

(g)

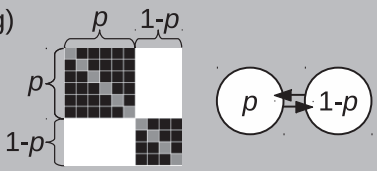

(h)

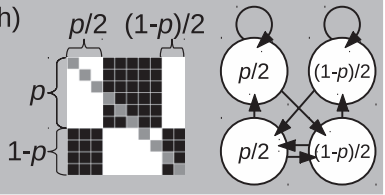

FIG. 1. Stationary states are shown, by the corresponding action matrices (left in the each panel) and the schematic diagram of clustering structure (right). Elements of action matrices are shown with white (cooperative) and black (not cooperative) pixels. Diagonal pixels are gray since they are not defined. Matrices shows are sorted to make their structure easier to see. Circles in schematic diagrams represents clusters of players. Arrows show players in which cluster are cooperative to players in which cluster. The fraction of the size of each cluster over total population is shown in each circle. The $p$ which appears in the fractions is the initial trust probability. These stationary states are analytically derived in Supplemental Material [15].

in a cluster are cooperative only within that cluster, we call the cluster an exclusive group. Among the eight states, only state (f) has multiple exclusive groups.

When the action rule is All-C, the stationary state is (a) irrespective of the assessment rule: all the players always cooperate with all the others in the end. And players always only defect each other in the stationary state [that corresponds to state (b)] if they adopt All-D for the action rule. Table I shows the stationary states in the case the action rule is DISC or pDISC. It is worth stressing that the most interesting stationary state (f), which has two exclusive groups in it, appears only when players adopt type-10 assessment rule.

The type-10 assessment rule, which is called Kandori [16] and hereafter denoted by $\mathrm{KN}$, is well studied. It is well known that KN promotes cooperation if players always share the same opinion, which is called public assessment [16-18]. It is also known that cooperation is less promoted by $\mathrm{KN}$ in private assessment [13,14]. However, it is first pointed out here that $\mathrm{KN}$ (and only $\mathrm{KN}$ ) cause the spontaneous split of players into multiple exclusive groups.

The essence of the derivation of the stationary states is as the following. Let us focus on the case that the action rule is DISC or pDISC, since it is trivial that the action rule All-C yields state (a) and All-D gives state (b). We first discuss the dynamics of opinion matrices and derive their stationary structure.

At the initial state, the opinions of any two players have no correlation, therefore disagreements of opinions on some 

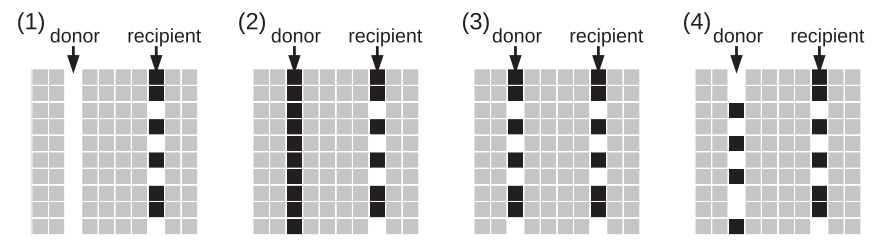

FIG. 2. Four possible opinion matrices after a revision. Elements of opinion matrices are shown with white (good) and black (bad) pixels. Elements irrelevant with the donor and the recipient do not affect, therefore they are shown with grey pixels. (1), (2), (3), and (4) correspond to the situation that all the players regard the donor as good, the all regard the donor as good, the all have the same opinion on the donor and on the recipient, and the all have the opposite opinions on the donor and on the recipient, respectively. Note that diagonal elements are also defined since they are not action matrices but opinion matrices.

players almost always exist between any two players. However, as we will see, agreements among at least part of players are formed in the repetition of the game.

Let us consider how the (partial) agreement is formed during a round. Each player observing a round revises his opinion on the donor according to the assessment rule, which is the function of his opinion on the recipient and the action of the donor. Since the action of the donor is observed by all players, only the difference in the opinion on the recipient can cause the difference in the new opinion on the donor. And some assessment rules, given an action of the donor, return the same reputation about the donor irrespective to the recipient's reputation. Therefore, the new opinions of observers on the donor (column in the opinion matrix in Fig. 2) after the round is either uniformly good or bad or the copy or inversion image of the observers' opinions on the recipient.

Under 12 out of 16 assessment rules, at least one of the actions of the donor (cooperation/defection) determines the new opinion of the observers irrespective to their opinion on the recipient. It means that the number of players on whom all players share the same opinion can increase. Once all players share the same opinion on all players, they never disagree about any players after the round. Therefore, players finally reach and are fixed to the state that all players share the same opinion on all players. In such cases, we say all players are in one opinion group [(1) of Fig. 3].

In contrast, with assessment rule 4, 7, $10(\mathrm{KN})$, and 13, only players who share the same opinion on the recipient share the same opinion on the donor. Because of this process, players who shared the same opinion on some players can begin to share the same opinion on other players (e.g., when a player on whom they shared the same opinion play a lot of rounds in series as the recipient). Finally, members who share the same opinion become same on all other players. In other words, players finally split into two opinion groups and players in different groups have the opposite opinions on all players. Note that the members of each of the opinion groups are the members who had the same opinion on a player at the initial round. At the initial state, every player is regarded as good by $p N$ players and as bad by $(1-p) N$ players on average. Therefore, the size ratio of the two opinion groups is always $p: 1-p$. Then, opinion matrices can be sorted as (2) of Fig. 3.

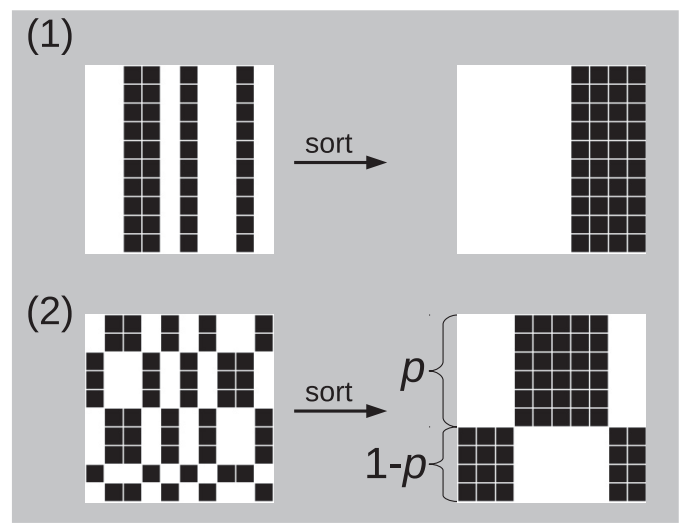

FIG. 3. Opinion matrices of players who form (1) one opinion group or (2) two opinion groups with size ratio $p: 1-p$. Opinion matrices are shown with white (good) and black (bad) pixels. Matrices in right hand side are results of sorted to make their structure easier to see.

Now we know whether all players belong to the same opinion group or they are split into two opinion groups with the size ratio $p: 1-p$, for each assessment rule. We can also determine the fraction of players who are regarded as good by themselves for each opinion group, at least if we fix the action rule. Consider the case the assessment rule is $\mathrm{KN}$ and the action rule is DISC. As mentioned above, assessment rule KN leads players to split into two opinion groups. A player cooperates with who is good in his opinion and defects against who is bad in his opinion because of the action rule DISC. In both cases, he and members of his opinion group will regard him as good because of the assessment rule KN. Therefore, the number of players who are good in their opinion monotonically increase. Finally, all players are good in their own opinion in both opinion groups. Similar procedures determine the stationary state of the opinion matrix for all combinations of an action rule and an assessment rule.

The stationary states of action matrices are clear from the action rules and the stationary states of opinion matrices. In the example of $\mathrm{KN}$ and DISC, players in each opinion group regard only those in the same group are good, therefore they cooperate with only the members of their opinion group. It directly means that they form two exclusive groups, with the size ratio $p: 1-p$, which is state (f). Other cases go similarly.

Until here, we assumed all players adopt a common action rule and revealed that players split into two exclusive groups when the assessment is $\mathrm{KN}$ and the action rule is DISC or pDISC. We finally study whether the state all players adopt DISC and the state all adopt pDISC are stable or not under the evolution of action rules, when the assessment rule is $\mathrm{KN}$. We consider the case that most of players adopt DISC (pDISC) and a slight fraction of players, "mutants", adopt another action rule and study whether the DISC players (pDISC players) get higher average payoff than the mutants. If DISC players (pDISC players) get higher average payoff, DISC (pDISC) is called an evolutionary stable strategy [19] and it suggests the mutants' action rule does not spread. Remember that $c$ is the cost which a donor takes to cooperate and $b$ is the benefit the recipient gains then. We proved that when the assessment rule is $\mathrm{KN}$, if and only if the benefit-cost ratio $b / c$ exceeds the 
threshold

$$
\left(\frac{b}{c}\right)^{*}=\frac{1}{2}\left[\frac{1}{(2 p-1)^{2}}+1\right],
$$

DISC and pDISC are evolutionary stable strategies [15]. The threshold rapidly increases as the initial trust probability $p$ approaches $1 / 2$. We will roughly see group separation is also accountable for this behavior of the threshold.

Consider the case that almost all players adopt DISC and a few mutants adopt All-D. All-D players hardly affect the relations among DISC players, therefore DISC players split into two opinion groups with the size ratio $p: 1-p$ as they do when DISC is the common action rule. DISC players are cooperative inside of each group and not cooperative across the groups. When the sizes of the two groups are more different, the chances for total DISC players to be matched inside of the groups increase, which means more chances of cooperation. At the same time, All-D players gain lower payoff when DISC player split with the larger size difference. Owing to the disagreement between the two groups, All-D players always have chances to receive cooperation from either opinion groups. If an All-D player defects against a player in an opinion group, the opinion group regards the All-D player as bad, however the other opinion group regards him as good. If two opinion groups' sizes have a very large difference, All-D players almost always defect against the members of the majority group, therefore most of All-D players can receive cooperation only from the small minority group. This is why DISC players' payoff can exceed All-D players' even with lower $b / c$, if $p$ is further from $1 / 2$. The case of pDISC goes similarly.

Our results show that a simple three-body interaction, which naturally comes from modeling agents' ability of monitoring the action between others, leads a novel type of self-organization into two groups. It is also interesting that the separation into groups is caused by mutual assessment, which usually plays a key role to overcome social dilemma. Especially, it is surprising and implicative that $\mathrm{KN}$, one of the best assessment rules to promote cooperation under public assessment, causes group separation under private assessment. This connection between the people's definitions of goodness and their group structure might be experimentally and empirically testable.

Extension of our model may enable us to understand not only two completely separated groups but also more complex and various group dynamics in our society. For example, if we consider more complex assessment rules, with which observers take their current opinion on the donor into consideration, perhaps more than two exclusive groups could be formed.

Formation of opinion groups plays the crucial role in the derivation of stationary states. This analysis based on opinion groups can be applied to the system with small error and the case assessment rules are different among players, which enables, for example, analysis of evolution of assessment rules.

In conclusion, it was revealed that players split into two exclusive groups by specific types of reciprocity norms and that the norms can be evolutionary stable. This can be an explanation of why people frequently form groups and cooperate only inside of the groups.
[1] G. Iniguez, J. Kertesz, K. K. Kaski, and R. A. Barrio, Phys. Rev. E 80, 066119 (2009).

[2] C. Gracia-Lázaro, L. F. Lafuerza, L. M. Floría, and Y. Moreno, Phys. Rev. E 80, 046123 (2009).

[3] L. Gauvin, J. P. Nadal, and J. Vannimenus, Phys. Rev. E 81, 066120 (2010)

[4] C. Castellano, S. Fortunato, and V. Loreto, Rev. Mod. Phys. 81, 591 (2009).

[5] M. A. Nowak, Science 314, 1560 (2006).

[6] M. A. Nowak and K. Sigmund, Nature 393, 573 (1998).

[7] H. Tajfel, M. G. Billig, R. P. Bundy, and C. Flament, Eur. J. Soc. Psychol. 1, 149 (1971).

[8] N. Masuda and H. Ohtsuki, Proc. R. Soc. Lond. B 274, 689 (2007).
[9] N. Masuda, J. Theor. Biol. 311, 8 (2012).

[10] M. Nakamura and N. Masuda, BMC Evol. Biol. 12, 1 (2012).

[11] H. Brandt and K. Sigmund, J. Theor. Biol. 231, 475 (2004).

[12] N. Takahashi and R. Mashima, J. Theor. Biol. 243, 418 (2006).

[13] S. Uchida, Phys. Rev. E 82, 036111 (2010).

[14] K. Sigmund, J. Theor. Biol. 299, 25 (2011).

[15] See Supplemental Material at http://link.aps.org/supplemental/ 10.1103/PhysRevE.87.030801 for the detail of the derivations.

[16] M. Kandori, Rev. Econ. Stud. 59, 63 (1992).

[17] H. Ohtsuki and Y. Iwasa, J. Theor. Biol. 231, 107 (2004).

[18] H. Ohtsuki and Y. Iwasa, J. Theor. Biol. 244, 518 (2006).

[19] M. J. Smith and G. R. Price, Nature 246, 15 (1973). 